\title{
The loss of the CD16 B73.1/Leu11c epitope occurring in some primary immunodeficiency diseases is not associated with the $\mathrm{Fc} \gamma \mathrm{RIIIa}-48 \mathrm{~L} / \mathrm{R} / \mathrm{H}$ polymorphism
}

\author{
MARZENA LENART ${ }^{1}$, ELZBIETA TRZYNA ${ }^{2}$, MAGDALENA RUTKOWSKA ${ }^{1}$, \\ KAROLINA BUKOWSKA-STRAKOVA ${ }^{1}$, ANNA SZAFLARSKA ${ }^{1}$, \\ ANNA PITUCH-NOWOROLSKA ${ }^{1}$, ANTONI SZCZEPANIK ${ }^{3}$, \\ MAREK ZEMBALA $^{1}$ and MACIEJ SIEDLAR ${ }^{1}$ \\ Departments of ${ }^{1}$ Clinical Immunology; ${ }^{2}$ Transplantation, Polish-American Institute of Pediatrics, \\ Jagiellonian University Medical College, Krakow; ${ }^{3}$ First Department of General and \\ Gastrointestinal Surgery, Jagiellonian University Medical College, Krakow, Poland
}

Received March 17,2010; Accepted May 20, 2010

DOI: $10.3892 /$ ijmm_00000483

\begin{abstract}
The loss of the CD16a, Fc receptor for IgG type III, (Fc $\gamma$ RIIIa) B73.1/Leu11c binding epitope, detected by the monoclonal antibody $(\mathrm{mAb})$ used in routine enumeration of NK cells or monocytes, has been observed in children with recurrent viral infections. It has also been linked with the change of leucine $(\mathrm{L})$ to histidine $(\mathrm{H})$ or arginine $(\mathrm{R})$ at amino acid position $48(\mathrm{Fc} \gamma \mathrm{RIII}-48 \mathrm{~L} / \mathrm{R} / \mathrm{H})$ in the CD16a receptor. The reactivity of the anti-CD16a clone B73.1/Leu11c mAb with monocytes and NK cells was examined in patients with primary immunodeficiencies $(n=167)$, gastrointestinal malignancies $(n=91)$ and healthy subjects $(n=88)$. Cells of only 12 children, 11 with diagnosed primary immunodeficiency and one with recurrent bacterial infections were not reactive with B73.1/Leu11c mAb. In contrast to previous findings, no linkage between the loss of B73.1/Leu11c binding epitope and herpes virus infections was observed. Furthermore, the sequence analysis of the FcrRIIIa gene performed in these 12 patients and 11 healthy subjects revealed that all of them had Fc $\gamma$ RIIIa-48L/L genotype. Thus, the loss of B73.1/Leu11c binding epitope was not associated with the Fc $\gamma$ RIIIa-48 polymorphism. The commonly described Fc $\gamma$ RIIIa-158 polymorphism was determined to be $158 \mathrm{~V} / \mathrm{V}$ in 11 patients and

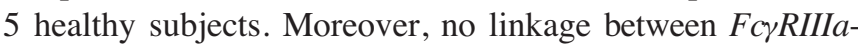
$48 \mathrm{~L} / \mathrm{L}$ and $-158 \mathrm{~F} / \mathrm{F}$ genotypes was observed. It is suggested that the loss of the B73.1/Leu11c binding epitope is connected with primary immunodeficiency disorders, but not associated with the Fc $\gamma$ RIIIa-48 polymorphism.
\end{abstract}

Correspondence to: Dr Maciej Siedlar, Department of Clinical Immunology Polish-American Institute of Pediatrics, Jagiellonian University Medical College, Wielicka 265, 30-663 Krakow, Poland E-mail: misiedla@cyf-kr.edu.pl

Key words: Fc receptor for IgG type III polymorphism, CD16a, B73.1/Leu11c monoclonal antibody

\section{Introduction}

The CD16 molecule, Fc receptor for IgG type III (Fc $\gamma \mathrm{RIII})$, is a glycoprotein expressed in NK cells, subpopulation of non-classical ('proinflammatory') $\mathrm{CD} 14^{+} \mathrm{CD} 16^{++}$monocytes (Fc $\gamma$ RIIIa), and neutrophils (Fc $\gamma$ RIIIb) (1-3). This receptor is engaged in antibody-dependent cellular cytotoxicity (ADCC) and plays a role in immunoregulation of proinflammatory response of leukocytes following binding of $\operatorname{IgG}(4-6)$. Several clones of anti-CD16 monoclonal antibodies (mAbs) are available for routine enumeration of NK cells and in immunophenotyping of proinflamatory monocytes. The mAbs include B73.1/Leu11c, GRM1, and PEN1 clones recognizing epitopes located at the first extracellular Ig-like domain, and the mAbs of 3G8, CLBFcRgran1, MEM-154 and Leu11a clones, recognizing epitopes at the membrane-proximal Ig-like domain in which the $\operatorname{IgG}$ binding site has been described $(7,8)$.

The gene coding Fc $\gamma$ RIIIa is very similar to a nearby gene coding Fc $\gamma$ RIIIb and both genes are located on chromosome 1 (9). Several polymorphisms of Fc $\gamma$ RIIIa have been characterized and two of them, associated with changes of amino acids at position 48 and 158, have been linked with higher susceptibility to viral infections, especially with Herpesviridae, and various $\mathrm{Fc}$ fragments of $\mathrm{IgG}$ binding capacity, respectively. The polymorphism at position 48 is located at the first extracellular Ig-like domain of Fc $\gamma$ RIIIa and results in the expression of either leucine (L), arginine (R) or histidine $(\mathrm{H})$, with gene frequencies in Caucasians of 86,6 and $8 \%$, respectively (10). The latter polymorphism, at amino acid position 158 , is located at the membrane-proximal domain and results in expression of the phenylalanine $(\mathrm{F})$ or valine $(\mathrm{V})(11)$. It was observed that the Fc $\gamma$ RIIIa-158F polymorphism is linked to $48 \mathrm{~L}$ isoform, while $158 \mathrm{~V}$ with $48 \mathrm{R}$ or $48 \mathrm{H}(10,12)$.

The polymorphism occurring at position 158 of the CD16 protein chain has been reported to influence the binding of $\mathrm{Fc}$ fragments of IgG by the receptor and has been connected with a higher risk of the development of autoimmune diseases $(10,11)$. The increased frequency of $158 \mathrm{~F} / \mathrm{F}$ isoform, which is 
a low IgG binding phenotype, was observed in patients with systemic lupus erythematosus (13-15), whereas a high IgG binding $158 \mathrm{~V} / \mathrm{V}$ isoform has been correlated with the higher risk of rheumatoid arthritis $(16,17)$. Moreover, studies on the association of certain Fc $\gamma$ RIIIa-158 isoforms with the efficiency of anti-CD20 mAbs therapy $(18,19)$ suggest that the presence of $158 \mathrm{~V} / \mathrm{V}$ or $158 \mathrm{~F} / \mathrm{V}$ phenotype predisposes to higher overall response to this therapy $(18,20,21)$. Eleven other described polymorphisms in the Fc $\gamma$ RIIII gene (Entrez Gene database, SNP for gene ID: 2214) were not connected with any specific clinical manifestations.

Several studies $(8,22-24)$ reported that the substitution of $\mathrm{L}$ by $\mathrm{R}$ or $\mathrm{H}$ at amino acid position 48 in the Fc $\gamma \mathrm{RIII}$ a receptor predisposes to recurrent viral infections of respiratory tract, herpes virus infections and low cytotoxic activity of NK cells. These reports suggest that the NK cells of patients with Fc $\gamma$ RIIIa-48R or $-48 \mathrm{H}$ did not react with anti-CD16 mAb of B73.1/Leu11c clone. Since the Fc $\gamma$ RIIIa-48R or $-48 H$ polymorphism was described to occur in a frequency of $14 \%$ in Caucasians (10), the rarity of reports of patients with NK cell defect (B73.1/Leu11c epitope loss) seems inexplicable, despite the common usage of the B73.1/Leu11c mAb in the immunophenotyping procedures of NK cells and monocyte subsets. Due to the predictable high frequency of the Fc $\gamma$ RIIIa-48 polymorphism, it is important to determine whether the Fc $\gamma$ RIIIa-48 polymorphism is indeed connected with the B73.1/Leu11c epitope loss. Recurrent viral infections, especially of the respiratory tract, are typical for patients with primary immunodeficiencies $(25,26)$. What is more, the frequent occurrence of recurrent viral infections in patients with immunodeficiency diseases suggest that it is necessary to determine the frequency of the occurrence of the B73.1/Leu11c epitope loss in these patients. Moreover, the decreased activity of NK cells is correlated with the higher risk of cancer development (27). Therefore, NK cell defect analysis appears to be important also in cases of patients with cancer. In this study we present the analysis of the occurrence of the B73.1/Leu11c epitope loss in patients with different primary immunodeficiency diseases and patients with gastrointestinal malignancies in comparison to age matched healthy control subjects. We also present genetic analysis of the entire coding region of $F c \gamma$ RIIIa gene performed in the group of patients lacking B73.1/Leu11c epitope and control group of healthy individuals whose NK cells and monocytes were reactive with B73.1/Leu11c mAbs.

\section{Materials and methods}

Patients. The patient group included: 167 patients with primary immunodeficiency diseases and 91 patients with gastrointestinal malignancies (Table I). As control, 88 healthy subjects were studied. Patients with immunodeficiency diseases were classified according to PAGID criteria (28). Individuals whose cells were not reactive with anti-CD16a B73.1/Leu11c mAbs were chosen for genetic studies of the FcyRIIIa gene. Their characteristics are presented in Table II. The genotyping of Fc $\gamma$ RIIIa gene was also performed in a group of 11 randomly chosen healthy control subjects with NK cells and monocytes that were reactive with anti-CD16a B73.1/Leu11c mAb. The Ethics Committee of the Jagiellonian
Table I. Characteristics of subjects included in the study.

\begin{tabular}{lr}
\hline Diagnosis & No. of subjects \\
\hline Children & \\
X-linked agammaglobulinemia & 11 \\
(XLA) & 41 \\
Selective IgA deficiency (sIgAD) & 31 \\
Transient hypogammaglobulinemia & \\
of infancy (THI) & 38 \\
Common variable immunodeficiency & \\
(CVID) & 7 \\
IgG subclasses deficiency & 1 \\
Immunodeficiency with thymoma & 1 \\
Aplastic anemia with & 13 \\
agammaglobulinemia & 3 \\
Unidentified hypogammaglobulinemias & 5 \\
Hyper IgM syndrome & 2 \\
Hyper IgE syndrome & 1 \\
Di George syndrome & \\
Unidentified defect of & 60 \\
cellular immunity & \\
Control group & \\
Adults & \\
CVID & \\
Gastrointestinal malignancies & \\
Gastric cancer & \\
Colorectal cancer & \\
Pancreatic cancer & \\
Control group & \\
\hline
\end{tabular}

University approved the study (KBET/107/B/2006). Informed consent was obtained from patients or children's parents.

Flow cytometry. Whole peripheral blood samples (100 $\mu 1)$ were drawn to EDTA containing tubes (Vacutest Kima, Arzergrande, Italy), incubated with mAbs: anti-CD3-PerCP (clone SK7, BD Biosciences), anti-CD19-PerCP (clone 4G7, BD Biosciences) or anti-CD14-APC (BD Biosciences Pharmingen) along with both clones of anti-CD16 mA: antiCD16, PE-conjugated (clone B73.1/Leu11c, BD Biosciences, San Jose, CA, USA) and FITC-conjugated (clone 3G8, BD Biosciences Pharmingen, San Diego, CA, USA) or with appropriate isotype-matched controls (BD Biosciences Pharmingen) for $20 \mathrm{~min}$ at $4^{\circ} \mathrm{C}$. Then erythrocytes were lysed with $1 \mathrm{ml}$ of FACS Lysing Solution (Becton Dickinson, Mountain View, CA, USA), washed twice and resuspended in PBS. Samples were analyzed in FACSCanto (Becton Dickinson Immunocytometry Systems, Palo Alto, CA, USA) flow cytometer using BD FACSDiva Software v6.1.2. The lymphocytes and monocytes were gated according to forward (FSC) and side side scatter (SSC) parameters.

Sequence analysis. DNA was extracted from whole peripheral blood samples using QIAmp DNA Mini Kit (Qiagen, Hilden, Germany). PCR was performed in a total volume of $20 \mu 1$, with $100 \mathrm{ng}$ DNA, $200 \mu \mathrm{M}$ of each dNTP, $0.5 \mathrm{U}$ AmpliTaq Gold polymerase (Applied Biosystems, Foster City, CA, 
Table II. Clinical characteristics of patients with the CD16 B73.1/Leu11c epitope loss.

\begin{tabular}{|c|c|c|c|}
\hline Patient & Age (years)/Gender & Diagnosis & Clinical features \\
\hline P1 & $2 / \mathrm{F}$ & THI & $\begin{array}{l}\text { recurrent upper and lower respiratory tract infections } \\
\text { (pharyngitis, laryngitis, otitis, bronchopneumonia), } \\
\text { infection with Klebsiella pneumoniae ESBL }\end{array}$ \\
\hline $\mathrm{P} 2$ & 7/M & sIgAD & $\begin{array}{l}\text { recurrent lower respiratory tract infections } \\
\text { (12 times pneumonia) }\end{array}$ \\
\hline P3 & $9 / \mathrm{F}$ & CVID & recurrent rhinopharyngitis, atopic dermatitis \\
\hline P4 & $7 / \mathrm{M}$ & CVID & $\begin{array}{l}\text { recurrent upper respiratory tract infections } \\
\text { (rhinopharyngitis, laryngitis), bronchial asthma }\end{array}$ \\
\hline P5 & $15 / \mathrm{M}$ & CVID & $\begin{array}{l}\text { recurrent Herpes simplex infections and stomatitis, } \\
\text { leukopenia, chronic sinusitis, Giardia lamblia infections }\end{array}$ \\
\hline P6 & $10 / \mathrm{M}$ & XLA & $\begin{array}{l}\text { recurrent upper and lower respiratory tract infections } \\
\text { (pharyngitis, bronchitis, chronic sinusitis, pneumonias), } \\
\text { Haemophilus influenzae in pharyngeal swabs }\end{array}$ \\
\hline P7 & $17 / \mathrm{M}$ & $\begin{array}{l}\text { immunodeficiency } \\
\text { with thymoma }\end{array}$ & $\begin{array}{l}\text { hepatitis type } \mathrm{C} \text {, recurrent respiratory tract infections, } \\
\text { miasthenia gravis, polyendocrynopathy, coeliac disease, } \\
\text { recurrent fungal infections of skin and mucosa }\end{array}$ \\
\hline P8 & $2 / \mathrm{M}$ & DiGeorge syndrome & recurrent bronchitis \\
\hline P9 & $13 / \mathrm{M}$ & DiGeorge syndrome & $\begin{array}{l}\text { recurrent upper respiratory tract infections } \\
\text { (rhinopharyngitis, laryngitis) and genitourinary tract } \\
\text { infections }\end{array}$ \\
\hline P10 & $11 / \mathrm{M}$ & $\begin{array}{l}\text { aplastic anemia, } \\
\text { agammaglobulinemia }\end{array}$ & $\begin{array}{l}\text { recurrent upper respiratory tract infections } \\
\text { (rhinopharyngitis, laryngitis, bronchitis, otitis), } \\
\text { before bone marrow transplantation }\end{array}$ \\
\hline P11 & $7 / \mathrm{M}$ & $\begin{array}{l}\text { undefined defect of cellular } \\
\text { immunity (decreased number } \\
\left.\text { of } \mathrm{CD}^{+} / \mathrm{CD}^{+} \text {lymphocytes }\right)\end{array}$ & $\begin{array}{l}\text { recurrent bronchitis and pneumonias, recurrent } \\
\text { genitourinary tract infections, teratoma in } \\
\text { newborn period, cerebral palsy, HIV-1 negative }\end{array}$ \\
\hline $\mathrm{P} 12$ & $13 / \mathrm{F}$ & not established & $\begin{array}{l}\text { recurrent sinusitis and tonsillitis, } \\
\text { Haemophilus influenzae and } \\
\text { Moraxella catarrhalis }\end{array}$ \\
\hline
\end{tabular}

USA) and $0.5 \mu \mathrm{M}$ of primers specific to each of five exons of FcRIIIa gene: exon 1, sense: 5'-GGC TGG GGA AAG GCT GTT TAC TT, antisense, 5'-CTG AAC CCA AGG CAT CTC AAA; exon2, sense, 5'-GCA AGA GGC ATG AAC AGT GGA G, antisense, 5'-CTG CTA ACC CCA CAT CAG CAT TT; exon 3, sense, 5'-CAC CAA GCA TGG GTT TGC AAT, antisense, 5'-AGT GGG ACC ACA CAT CAT CTC AT; exon 4, sense, 5'-TGC AGG GTT GAC TCC CAA TCT, antisense, 5'-CCA ACT CAA CTT CCC AGT GTG ATT; exon 5, sense, 5'-GGT GAG CTG TCC TCT GCT CAG ATA, antisense, 5'-GAA ATG TTC AGA GAT GCT GCT GCT (TIB MolBiol, Poznan, Poland), as described by Treon et al (20). The thermocycling conditions were $10 \mathrm{~min}$ at $95^{\circ} \mathrm{C}$ followed by 35 cycles at $95^{\circ} \mathrm{C}$ for $15 \mathrm{sec}$, specific for each set of primers temperature of annealing (exon $1,55^{\circ} \mathrm{C} ; 2,55^{\circ} \mathrm{C}$; $3,56^{\circ} \mathrm{C} ; 4,54^{\circ} \mathrm{C} ; 5,58^{\circ} \mathrm{C}$ ) for $30 \mathrm{sec}, 72^{\circ} \mathrm{C}$ for $30 \mathrm{sec}$, followed by final elongation for $10 \mathrm{~min}$ at $72^{\circ} \mathrm{C}$, then held in $4{ }^{\circ} \mathrm{C}$. The PCR products were purified with a QIAquick PCR Purification Kit (Qiagen). Sequencing PCR was performed on the PCR products using exon-specific primer and Big Dye Terminator v3.1 Cycle Sequencing Kit (Applied Biosystems). The PCR assay was performed with $10 \mathrm{ng}$ DNA, $8 \mu \mathrm{l}$ Terminator Ready Reaction Mix, 3.2 pM of primer, described above, in a total volume of $20 \mu 1$ and reaction was performed by 25 amplification cycles, $95^{\circ} \mathrm{C}$ for $10 \mathrm{sec}, 50^{\circ} \mathrm{C}$ for $5 \mathrm{sec}$, $60^{\circ} \mathrm{C}$ for $4 \mathrm{~min}$, then held at $4^{\circ} \mathrm{C}$. PCR products were purified by ethanol/EDTA precipitation and dissolved in $25 \mu 1$ of deionized formamide (Applied Biosystems), denatured at $95^{\circ} \mathrm{C}$ for $3 \mathrm{~min}$, and run on the Applied Biosystems 310 sequencing apparatus and analyzed with the Sequencing Analysis software (Applied Biosystems). For each exon the bidirectional sequence analysis was performed. The DNA sequencing results were aligned to the Fc $\gamma$ RIIII gene sequence available in the Entrez Gene database (gene ID: 2214). In accordance to commonly used enumeration of Fc $\gamma$ RIIIa polymorphisms $(10,12,20)$ the first nucleotide of the first codon for the first amino acid of the first extracellular domain was designated as nucleotide 1 .

\section{Results}

Expression of Fc $\gamma$ RIIIa on monocytes and NK cells. Peripheral blood lymphocytes and monocytes from patients with immunodeficiency diseases, cancer patients or healthy subjects were analyzed using two different clones of antiCD16 mAbs, B73.1/Leu11c and 3G8 recognizing distinct epitopes of the Fc $\gamma$ RIIIa receptor (29). NK cell population was determined as CD3-CD19- cells in a lymphocytic gate, while monocytes were determined according to FSC and SSC parameters and CD14 positivity of the cells (Fig. 1). The NK cells and monocytes of 11 pediatric patients with diagnosed primary immunodeficiency and one child with recurrent 

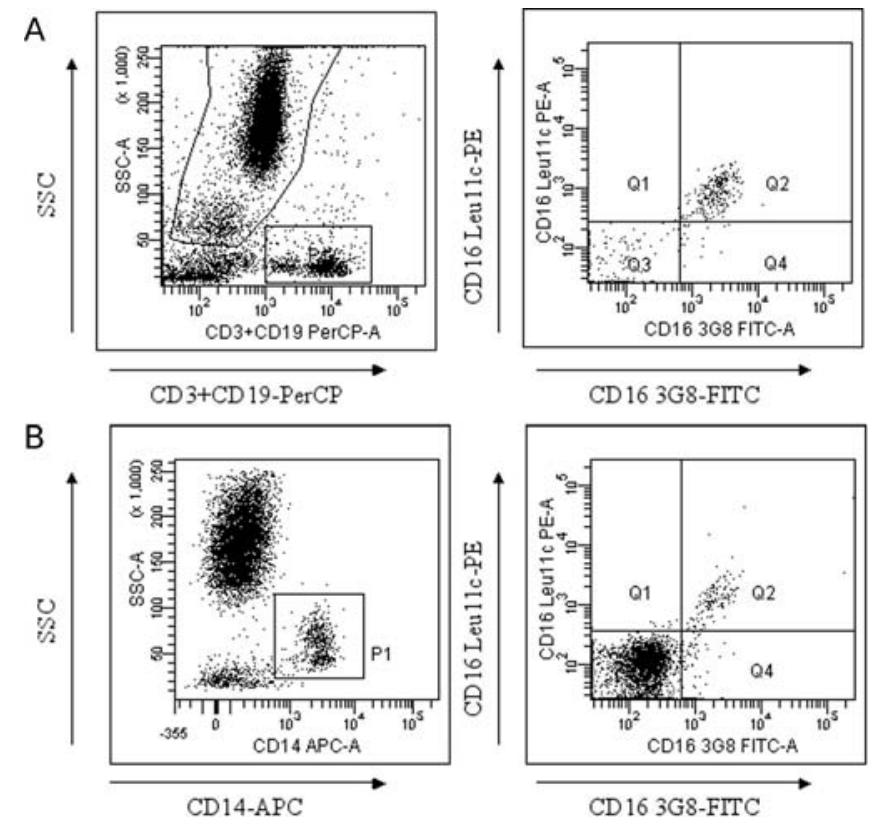

Figure 1. Flow cytometry analysis of the expression of CD16 B73.1/Leu11c and 3G8 epitopes on NK cells and monocytes. (A) NK cells were determined as CD3-CD19- cells in lymphocytic gate. (B) Monocytes were determined as $\mathrm{CD} 14^{+}$cells.

infections were reactive only with the anti-CD16 3G8, but not with the B73.1/Leu11c mAbs, while the other analyzed patients and healthy subjects were reactive with both mAbs (Fig. 2). These 12 patients (Table II) and 11 randomly chosen healthy subjects, whose B73.1/Leu11c mAb binding was not disturbed, were chosen for the genetic studies of the Fc $\gamma$ RIIIa gene polymorphisms.

Sequence analysis of Fc $\gamma$ RIIIa gene coding region. Sequence analysis of the entire coding region of Fc $\gamma$ RIIII gene (Table III) of the first, second and fifth exon did not reveal any nucleotide changes in the analyzed individuals in comparison to the reference sequence. However, within third and fourth exons, four different single nucleotide polymorphisms were detected, both in patients as well as the control group. Polymorphisms are summarized for each patient in Table III.

Within the third exon the Fc $\gamma$ RIIIa-48R or $48 \mathrm{H}$ isoforms, correlated with the B73.1/Leu11c epitope loss and higher susceptibility to viral infections, was not observed in any of the analyzed patients or healthy control subjects, due to the detected presence of $\mathrm{Fc} \gamma \mathrm{RIIIa}-48 \mathrm{~L} / \mathrm{L}$ isoform as only thymine (T) was detected at nucleotide position 230 (Fig. 3). The nucleotide change of adenine (A) to guanine $(\mathrm{G})$ within the third exon of Fc $\gamma$ RIIIa at nucleotide position 346, resulting in isoleucine (I) to $\mathrm{V}$ change at amino acid position 88 , was detected in 2 patients (P2 and 11) and in 3 control subjects $(\mathrm{C} 2,8,10)$ and all of them were $\mathrm{Fc} \gamma$ RIIIa-88I/V heterozygotes.

Within the fourth exon of the FcrRIIIa gene three polymorphisms were detected, at amino acid positions 129, 137 and 158. The nucleotide change of $\mathrm{G}$ to $\mathrm{A}$ at position 440 resulting in amino acid change of glycine (G) to aspartic acid (D) at position 129 was detected in patient 11 who was FcyRIIIa129D/D homozygote. Patients 4, 11 and 12 had Fc $\gamma$ RIIIa$137 \mathrm{H} / \mathrm{H}$ variation due to $\mathrm{T}$ to cytosine (C) substitution at
A
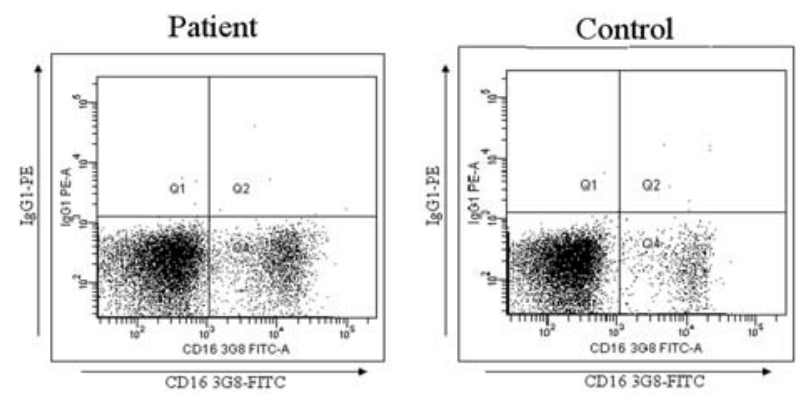

B
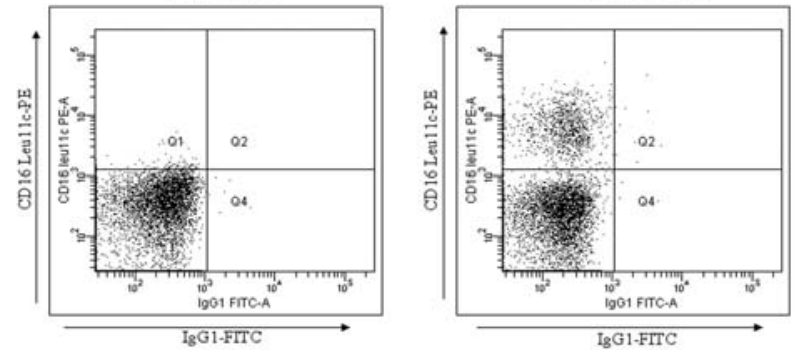

C
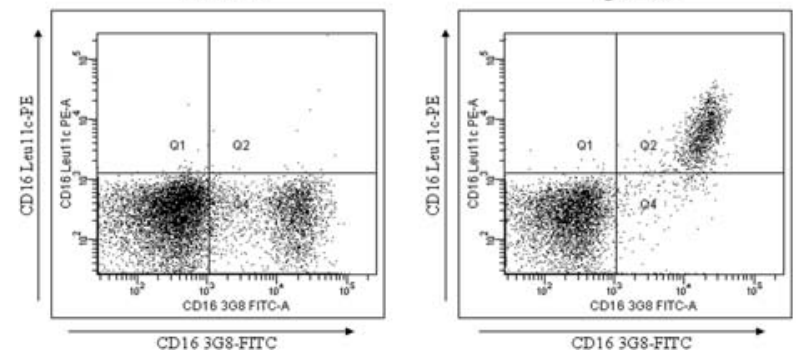

Figure 2. Flow cytometry analysis of the expression of CD16 B73.1/Leu11c and 3G8 epitopes on the monocytes and NK cells of one of the patients and healthy control subjects. The monocytes and NK cells were gated on FSC and SSC parameters. Staining pattern of mAbs (A) anti-CD16 3G8-FITC and IgG1-FITC (B) anti-CD16 B73.1/Leu11c-PE and IgG1-PE and (C) antiCD16 3G8-FITC and B73.1/Leu11c-PE.

Patient

Control
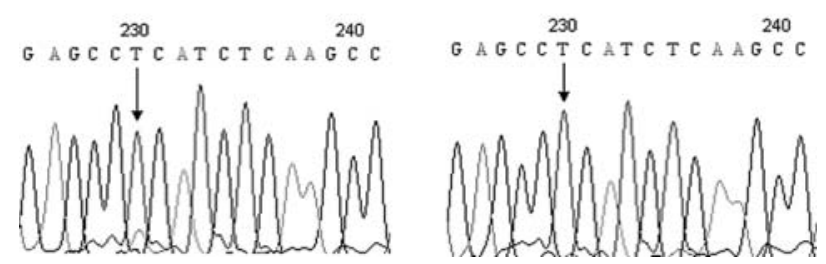

Figure 3. The sequence of the fragment of the third exon of the $\mathrm{Fc} \gamma \mathrm{RIII}$ gene of one patient and control subject showing nucleotide 230 coding 48 amino acid, which substitution was correlated with the CD16 B73.1/Leu11c epitope loss. Sequence fragment displayed by Chromas Litesoftware.

nucleotide position 472 and tyrosine (Y) to $\mathrm{H}$ change. In the control subjects, none had the Fc $\gamma$ RIIIa-129 or -137 polymorphism detected. The Fc $\gamma$ RIIIa-158 isoform was detected in 11 out of 12 analyzed patients and 5 out of 11 control subjects, since single nucleotide change of $\mathrm{T}$ to $\mathrm{G}$ at position 526 was confirmed, resulting in Fc $\gamma$ RIIIa-158V/V isoform. However, Fc $\gamma$ RIIIa-158 isoform was indeterminable in 1 patient (P10) and 6 control subjects (C5-8, 10 and 11). Those individuals had both $\mathrm{T}$ and $\mathrm{G}$ nucleotide detected at nucleotide position 526. The Fc $\gamma$ RIIIa-158 polymorphism was not confirmed in 7 analyzed subjects since two peaks of the same 
Table III. Summary of detected polymorphisms and heterozygotic isoforms with predicted amino acids in the Fc $\gamma$ RIIIa gene.

\begin{tabular}{|c|c|c|}
\hline & Polymorphisms detected & Heterozygotic isoforms detected with predicted amino acids \\
\hline \multicolumn{3}{|c|}{ Patient no. } \\
\hline $\mathrm{P} 1$ & 48L/L; $158 \mathrm{~V} / \mathrm{V}$ & 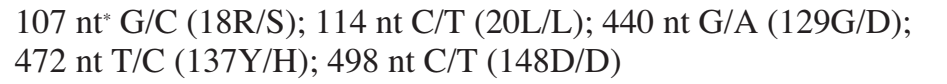 \\
\hline $\mathrm{P} 2$ & $48 \mathrm{~L} / \mathrm{L} ; 88 \mathrm{I} / \mathrm{V} ; 158 \mathrm{~V} / \mathrm{V}$ & $\begin{array}{l}440 \mathrm{nt} \mathrm{G} / \mathrm{A}(129 \mathrm{G} / \mathrm{D}) ; 472 \mathrm{nt} \mathrm{T/C}(137 \mathrm{Y} / \mathrm{H}) \\
498 \mathrm{nt} \mathrm{C} / \mathrm{T}(148 \mathrm{D} / \mathrm{D})\end{array}$ \\
\hline P3 & $48 \mathrm{~L} / \mathrm{L} ; 158 \mathrm{~V} / \mathrm{V}$ & $\begin{array}{l}107 \mathrm{nt} \mathrm{G} / \mathrm{C}(18 \mathrm{R} / \mathrm{S}) ; 114 \mathrm{nt} \mathrm{C} / \mathrm{T}(20 \mathrm{~L} / \mathrm{L}) ; 274 \mathrm{nt} \mathrm{G} / \mathrm{A}(64 \mathrm{D} / \mathrm{N}) \\
440 \mathrm{nt} \mathrm{G} / \mathrm{A}(129 \mathrm{G} / \mathrm{D}) ; 472 \mathrm{nt} \text { T/C }(137 \mathrm{Y} / \mathrm{H}) ; 498 \mathrm{nt} \mathrm{C} / \mathrm{T}(148 \mathrm{D} / \mathrm{D})\end{array}$ \\
\hline $\mathrm{P} 4$ & 48L/L; 137H/H; 158V/V & $\begin{array}{l}107 \mathrm{nt} \mathrm{G} / \mathrm{C}(18 \mathrm{R} / \mathrm{S}) ; 114 \mathrm{nt} \mathrm{C} / \mathrm{T}(20 \mathrm{~L} / \mathrm{L}) \\
440 \mathrm{nt} \mathrm{G} / \mathrm{A}(129 \mathrm{G} / \mathrm{D}) ; 498 \mathrm{nt} \mathrm{C} / \mathrm{T}(148 \mathrm{D} / \mathrm{D})\end{array}$ \\
\hline P5 & $48 \mathrm{~L} / \mathrm{L} ; 158 \mathrm{~V} / \mathrm{V}$ & $440 \mathrm{nt} \mathrm{G} / \mathrm{A}(129 \mathrm{G} / \mathrm{D}) ; 472 \mathrm{nt} \mathrm{T} / \mathrm{C}(137 \mathrm{Y} / \mathrm{H}) ; 498 \mathrm{nt} \mathrm{C} / \mathrm{T}(148 \mathrm{D} / \mathrm{D})$ \\
\hline P6 & $48 \mathrm{~L} / \mathrm{L} ; 158 \mathrm{~V} / \mathrm{V}$ & $440 \mathrm{nt} \mathrm{G} / \mathrm{A}(129 \mathrm{G} / \mathrm{D}) ; 472 \mathrm{nt} \mathrm{T} / \mathrm{C}(137 \mathrm{Y} / \mathrm{H}) ; 498 \mathrm{nt} \mathrm{C} / \mathrm{T}(148 \mathrm{D} / \mathrm{D})$ \\
\hline P7 & $48 \mathrm{~L} / \mathrm{L} ; 158 \mathrm{~V} / \mathrm{V}$ & $\begin{array}{l}107 \mathrm{nt} \mathrm{G} / \mathrm{C}(18 \mathrm{R} / \mathrm{S}) ; 114 \mathrm{nt} \mathrm{C} / \mathrm{T}(20 \mathrm{~L} / \mathrm{L}) ; 440 \mathrm{nt} \mathrm{G} / \mathrm{A}(129 \mathrm{G} / \mathrm{D}) \\
472 \mathrm{nt} \mathrm{T/C}(137 \mathrm{Y} / \mathrm{H}) ; 498 \mathrm{nt} \mathrm{C} / \mathrm{T}(148 \mathrm{D} / \mathrm{D})\end{array}$ \\
\hline P8 & $48 \mathrm{~L} / \mathrm{L} ; 158 \mathrm{~V} / \mathrm{V}$ & $\begin{array}{l}107 \mathrm{nt} \mathrm{G} / \mathrm{C}(18 \mathrm{R} / \mathrm{S}) ; 114 \mathrm{nt} \mathrm{C} / \mathrm{T}(20 \mathrm{~L} / \mathrm{L}) ; 440 \mathrm{nt} \mathrm{G} / \mathrm{A}(129 \mathrm{G} / \mathrm{D}) \\
472 \mathrm{nt} \mathrm{T/C}(137 \mathrm{Y} / \mathrm{H}) ; 498 \mathrm{nt} \mathrm{C} / \mathrm{T}(148 \mathrm{D} / \mathrm{D})\end{array}$ \\
\hline P9 & $48 \mathrm{~L} / \mathrm{L} ; 158 \mathrm{~V} / \mathrm{V}$ & $\begin{array}{l}107 \mathrm{nt} \mathrm{G} / \mathrm{C}(18 \mathrm{R} / \mathrm{S}) ; 114 \mathrm{nt} \mathrm{C} / \mathrm{T}(20 \mathrm{~L} / \mathrm{L}) ; 274 \mathrm{nt} \mathrm{G} / \mathrm{A}(64 \mathrm{D} / \mathrm{N}) ; 4 \\
40 \mathrm{nt} \mathrm{G} / \mathrm{A}(129 \mathrm{G} / \mathrm{D}) ; 472 \mathrm{nt} \mathrm{T/C} \mathrm{(137Y/H);} 498 \mathrm{nt} \mathrm{C/T} \mathrm{(148D/D)}\end{array}$ \\
\hline $\mathrm{P} 10$ & $\begin{array}{l}\text { 48L/L; } 158 \text { amino acid } \\
\text { undetermined }\end{array}$ & $\begin{array}{l}440 \mathrm{nt} \mathrm{G} / \mathrm{A}(129 \mathrm{G} / \mathrm{D}) ; 472 \mathrm{nt} \mathrm{T/C}(137 \mathrm{Y} / \mathrm{H}) \\
498 \mathrm{nt} \mathrm{C} / \mathrm{T}(148 \mathrm{D} / \mathrm{D}) ; 526 \mathrm{nt} \mathrm{T/G}(158 \mathrm{~F} / \mathrm{V})\end{array}$ \\
\hline P11 & $\begin{array}{l}48 \mathrm{~L} / \mathrm{L} ; 88 \mathrm{I} / \mathrm{V} ; 129 \mathrm{D} / \mathrm{D} ; \\
137 \mathrm{H} / \mathrm{H} ; 158 \mathrm{~V} / \mathrm{V}\end{array}$ & 114 nt C/T (20L/L); 498 nt C/T (148D/D) \\
\hline $\mathrm{P} 12$ & 48L/L; 137H/H; 158V/V & $\begin{array}{l}107 \mathrm{nt} \mathrm{G} / \mathrm{C}(18 \mathrm{R} / \mathrm{S}) ; 114 \mathrm{nt} \mathrm{C/T} \mathrm{(20L/L);} 274 \mathrm{nt} \mathrm{G/A} \mathrm{(64D/N);} \\
440 \mathrm{nt} \mathrm{G/A} \mathrm{(129G/D);} 498 \mathrm{nt} \mathrm{C/T} \mathrm{(148D/D)}\end{array}$ \\
\hline \multicolumn{3}{|c|}{ Control no. } \\
\hline $\mathrm{C} 1$ & $48 \mathrm{~L} / \mathrm{L} ; 158 \mathrm{~V} / \mathrm{V}$ & $440 \mathrm{nt} \mathrm{G} / \mathrm{A}(129 \mathrm{G} / \mathrm{D}) ; 472 \mathrm{nt} \mathrm{T} / \mathrm{C}(137 \mathrm{Y} / \mathrm{H}) ; 498 \mathrm{nt} \mathrm{C} / \mathrm{T}(148 \mathrm{D} / \mathrm{D})$ \\
\hline $\mathrm{C} 2$ & $48 \mathrm{~L} / \mathrm{L} ; 88 \mathrm{I} / \mathrm{V} ; 158 \mathrm{~V} / \mathrm{V}$ & $440 \mathrm{nt} \mathrm{G} / \mathrm{A}(129 \mathrm{G} / \mathrm{D}) ; 472 \mathrm{nt} \mathrm{T} / \mathrm{C}(137 \mathrm{Y} / \mathrm{H}) ; 498 \mathrm{nt} \mathrm{C} / \mathrm{T}(148 \mathrm{D} / \mathrm{D})$ \\
\hline $\mathrm{C} 3$ & $48 \mathrm{~L} / \mathrm{L} ; 158 \mathrm{~V} / \mathrm{V}$ & $\begin{array}{l}107 \mathrm{nt} \mathrm{G} / \mathrm{C}(18 \mathrm{R} / \mathrm{S}) ; 114 \mathrm{nt} \mathrm{C} / \mathrm{T}(20 \mathrm{~L} / \mathrm{L}) ; 194 \mathrm{nt} \mathrm{G} / \mathrm{A}(47 \mathrm{~S} / \mathrm{N}) \\
440 \mathrm{nt} \mathrm{G} / \mathrm{A}(129 \mathrm{G} / \mathrm{D}) ; 472 \mathrm{nt} \text { T/C }(137 \mathrm{Y} / \mathrm{H}) ; 498 \mathrm{nt} \mathrm{C} / \mathrm{T}(148 \mathrm{D} / \mathrm{D})\end{array}$ \\
\hline $\mathrm{C} 4$ & $48 \mathrm{~L} / \mathrm{L} ; 158 \mathrm{~V} / \mathrm{V}$ & $\begin{array}{l}107 \mathrm{nt} \mathrm{G} / \mathrm{C}(18 \mathrm{R} / \mathrm{S}) ; 114 \mathrm{nt} \mathrm{C} / \mathrm{T}(20 \mathrm{~L} / \mathrm{L}) ; 440 \mathrm{nt} \mathrm{G} / \mathrm{A}(129 \mathrm{G} / \mathrm{D}) \\
472 \mathrm{nt} \mathrm{T/C}(137 \mathrm{Y} / \mathrm{H}) ; 498 \mathrm{nt} \mathrm{C} / \mathrm{T}(148 \mathrm{D} / \mathrm{D})\end{array}$ \\
\hline $\mathrm{C} 5$ & $\begin{array}{l}\text { 48L/L; } 158 \text { amino acid } \\
\text { undetermined }\end{array}$ & $\begin{array}{l}440 \mathrm{nt} \mathrm{G/A}(129 \mathrm{G} / \mathrm{D}) ; 472 \mathrm{nt} \mathrm{T} / \mathrm{C}(137 \mathrm{Y} / \mathrm{H}) \\
498 \mathrm{nt} \mathrm{C} / \mathrm{T}(148 \mathrm{D} / \mathrm{D}) ; 526 \mathrm{nt} \mathrm{T} / \mathrm{G}(158 \mathrm{~F} / \mathrm{V})\end{array}$ \\
\hline C6 & $\begin{array}{l}\text { 48L/L; } 158 \text { amino acid } \\
\text { undetermined }\end{array}$ & $\begin{array}{l}440 \mathrm{nt} \mathrm{G} / \mathrm{A}(129 \mathrm{G} / \mathrm{D}) ; 472 \mathrm{nt} \mathrm{T} / \mathrm{C}(137 \mathrm{Y} / \mathrm{H}) \\
498 \mathrm{nt} \mathrm{C} / \mathrm{T}(148 \mathrm{D} / \mathrm{D}) ; 526 \mathrm{nt} \mathrm{T/G}(158 \mathrm{~F} / \mathrm{V})\end{array}$ \\
\hline $\mathrm{C} 7$ & $\begin{array}{l}\text { 48L/L; } 158 \text { amino acid } \\
\text { undetermined }\end{array}$ & $\begin{array}{l}440 \mathrm{nt} \mathrm{G} / \mathrm{A}(129 \mathrm{G} / \mathrm{D}) ; 472 \mathrm{nt} \mathrm{T} / \mathrm{C}(137 \mathrm{Y} / \mathrm{H}) \\
498 \mathrm{nt} \mathrm{C} / \mathrm{T}(148 \mathrm{D} / \mathrm{D}) ; 526 \mathrm{nt} \mathrm{T/G}(158 \mathrm{~F} / \mathrm{V})\end{array}$ \\
\hline $\mathrm{C} 8$ & $\begin{array}{l}\text { 48L/L; 88I/V; } 158 \text { amino acid } \\
\text { undetermined }\end{array}$ & $\begin{array}{l}194 \mathrm{nt} \mathrm{G} / \mathrm{A}(47 \mathrm{~S} / \mathrm{N}) ; 440 \mathrm{nt} \mathrm{G} / \mathrm{A}(129 \mathrm{G} / \mathrm{D}) ; 472 \mathrm{nt} \mathrm{T/C}(137 \mathrm{Y} / \mathrm{H}) \\
498 \mathrm{nt} \mathrm{C} / \mathrm{T}(148 \mathrm{D} / \mathrm{D}) ; 526 \mathrm{nt} \mathrm{T/G}(158 \mathrm{~F} / \mathrm{V})\end{array}$ \\
\hline C9 & $48 \mathrm{~L} / \mathrm{L} ; 158 \mathrm{~V} / \mathrm{V}$ & $\begin{array}{l}107 \text { nt G/C (18R/S); } 114 \text { nt C/T (20L/L); } 440 \text { nt G/A (129G/D); } \\
472 \text { nt T/C (137Y/H); } 498 \text { nt C/T (148D/D) }\end{array}$ \\
\hline $\mathrm{C} 10$ & $\begin{array}{l}48 \mathrm{~L} / \mathrm{L} ; 88 \mathrm{I} / \mathrm{V} ; 158 \text { amino acid } \\
\text { undetermined }\end{array}$ & $\begin{array}{l}194 \mathrm{nt} \mathrm{G} / \mathrm{A}(47 \mathrm{~S} / \mathrm{N}) ; 440 \mathrm{nt} \mathrm{G} / \mathrm{A}(129 \mathrm{G} / \mathrm{D}) ; 472 \mathrm{nt} \mathrm{T/C}(137 \mathrm{Y} / \mathrm{H}) \\
498 \mathrm{nt} \mathrm{C} / \mathrm{T}(148 \mathrm{D} / \mathrm{D}) ; 526 \mathrm{nt} \mathrm{T/G}(158 \mathrm{~F} / \mathrm{V})\end{array}$ \\
\hline $\mathrm{C} 11$ & $\begin{array}{l}48 \mathrm{~L} / \mathrm{L} ; 158 \text { amino acid } \\
\text { indetermined }\end{array}$ & $\begin{array}{l}107 \mathrm{nt} \mathrm{G} / \mathrm{C}(18 \mathrm{R} / \mathrm{S}) ; 114 \mathrm{nt} \mathrm{C} / \mathrm{T}(20 \mathrm{~L} / \mathrm{L}) ; 440 \mathrm{nt} \mathrm{G} / \mathrm{A}(129 \mathrm{G} / \mathrm{D}) ; \\
472 \mathrm{nt} \mathrm{T} / \mathrm{C}(137 \mathrm{Y} / \mathrm{H}) ; 498 \mathrm{nt} \mathrm{C} / \mathrm{T}(148 \mathrm{D} / \mathrm{D}) ; 526 \mathrm{nt} \mathrm{T} / \mathrm{G}(158 \mathrm{~F} / \mathrm{V})\end{array}$ \\
\hline
\end{tabular}

*nt, nucleotide

height on the electropherogram were detected at nucleotide position 526, one for $\mathrm{T}$ and one for $\mathrm{G}$. It was not possible to establish whether this is due to the heterozygotic isoform of

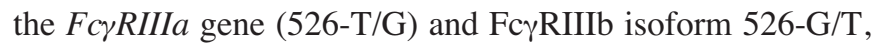
or due to homozygotic isoforms of both genes, 526-T/T and 526-G/G, respectively. That is why, in case of these subjects, the amino acid position 158 polymorphism in the Fc $\gamma$ RIIIa receptor was marked as indeterminable.
Seven other Fc $\mathrm{RIII}$ a heterozygotic isoforms were noted in the third and fourth exons (at nucleotide positions 107, $114,194,274,440,472$ and 498). In the fourth exon in all patients and control subjects $\mathrm{C} / \mathrm{T}$ heterozygotic isoform at nucleotide position 498 (amino acid position 148) was detected. However, the $\mathrm{C}$ to $\mathrm{T}$ change of nucleotide position $498 \mathrm{did}$ not change the amino acid position 148, so the Fc $\gamma$ RIIIa-148D/D phenotype was predicted in all analyzed individuals. Twenty 
a
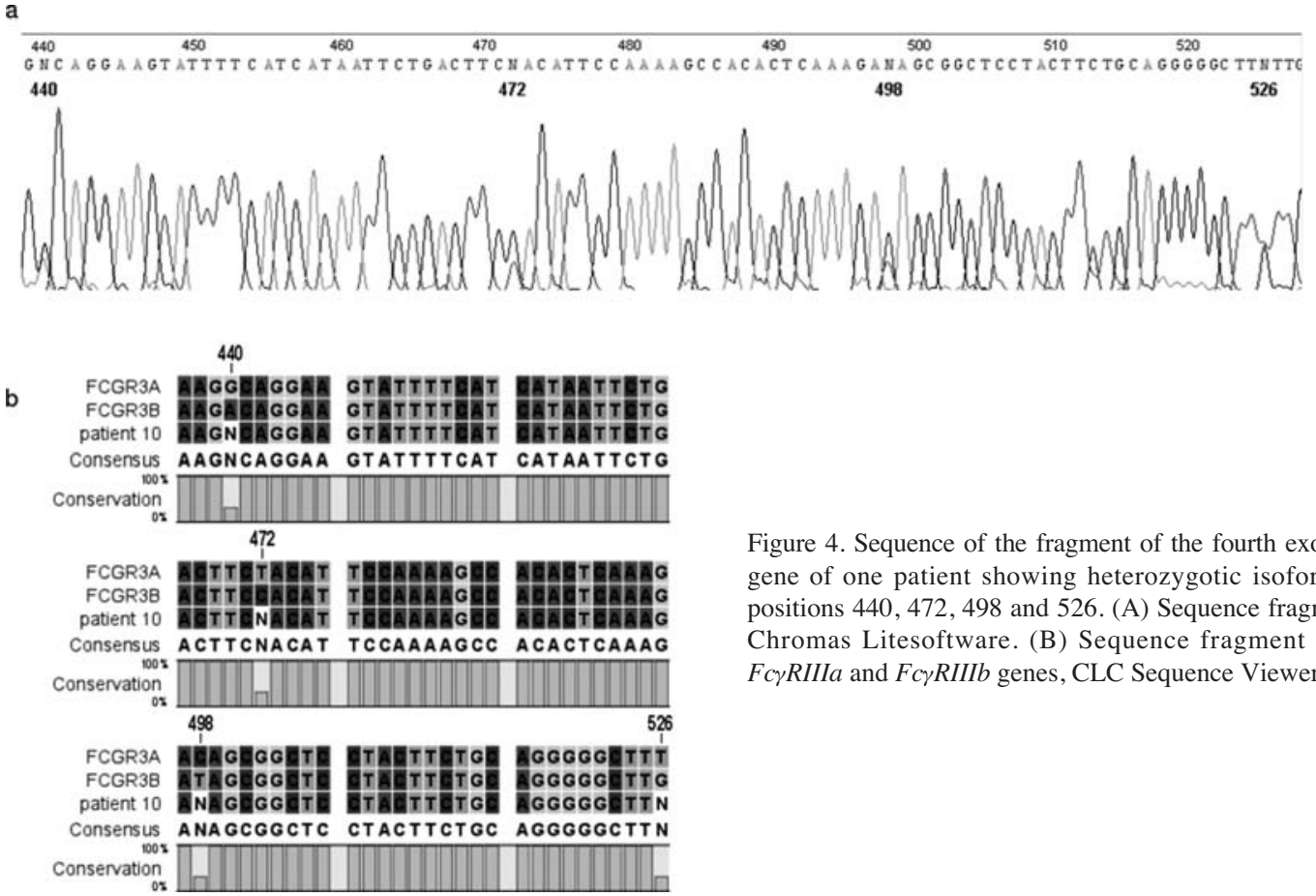

subjects, except patients $4,11,12$, had T/C genotype at nucleotide position 472 (amino acid position 137) and $\mathrm{T}$ at position 472 results in $\mathrm{Y}$ expression, while $\mathrm{C}$ results in $\mathrm{H}$. All analyzed individuals, except patient 11, had 440-G/A heterozygotic genotype detected, resulting in the Fc $\gamma$ RIIIa-129G/D phenotype predicted. Within the third exon of the Fc $\gamma$ RIIIa gene in 8 patients $(\mathrm{P} 1,3,4,7,8,9,11,12)$ and 4 control subjects $(\mathrm{C} 3,4,9,11)$ the heterozygotic isoforms $\mathrm{G} / \mathrm{C}$ and $\mathrm{C} / \mathrm{T}$ were observed at nucleotide positions 107 (amino acid position 18) and 114 (amino acid position 20), respectively. The substitution of $\mathrm{G}$ by $\mathrm{C}$ at nucleotide 107 results in the expression of serine $(S)$ instead of $R$, while the presence of $T$ at position 114 did not alter the amino acid position 20 and the Fc $\gamma$ RIIIa-20L/L isoform was predicted. At 194 nucleotide, amino acid position 47 , three healthy control subjects $(\mathrm{C} 3,8$, 10), had G and A detected while the rest of analyzed individuals had only $\mathrm{G}$ determined. The presence of $\mathrm{G}$ at nucleotide position 194 results in $\mathrm{S}$ expression, while A results in asparagine $(\mathrm{N})$. In 3 patients $(\mathrm{P} 3,9,12)$ heterozygotic $\mathrm{G} / \mathrm{A}$ isoform was detected at nucleotide position 274 , amino acid position 64 and $\mathrm{G}$ results in $\mathrm{D}$ expression, while $\mathrm{A}$ in $\mathrm{N}$ expression.

\section{Discussion}

There are several reports describing children with low cytotoxic activity of NK cells and similar clinical characteristics, recurrent infections, mostly with Herpesviridae (8,22-24). These reports indicate that higher susceptibility to viral infection correlates with the NK cell defects that manifest as a loss of the CD16 B73.1/Leu11c epitope. The epitope loss was described to be caused by the change of the amino acid position 48 from $\mathrm{L}$ to $\mathrm{R}$ or $\mathrm{H}$. However, the more recent reports described the amino acid position 48 to be polymorphic also in the healthy individuals, since $\mathrm{R}$ or $\mathrm{H}$ expression occurs in Caucasians with the frequency of $\sim 14 \%$ (10). That is why, the common usage of the anti-CD16 B73.1/Leu11c mAbs in routine immunodiagnostics should provide data on many more patients with NK cell defect. Therefore, it seems questionable that this rarely occurring defect is connected with the frequent polymorphism. Furthermore, the rarity of the studies reporting the subjects with recurrent viral infections and the Fc $\gamma$ RIIIa-48R/H polymorphism seems intriguing. Due to the higher frequency of viral infections in patients with primary immunodeficiency diseases and the connection of NK cell activity with cancer development, in this study we present the analysis of the frequency of CD16 B73.1/Leu11c epitope loss on NK cells and monocytes in a large group of patients with primary immunodeficiencies and patients with gastrointestinal malignancies.

From an entire group of analyzed individuals, the B73.1/ Leu11c epitope loss was detected only in 12 children, including 11 children with primary immunodeficiency diseases and one child with recurrent respiratory tract infections, but was not detected in patients with gastrointestinal malignancies and healthy subjects. According to the allele frequency provided by de Haas et al (10) in our group of 346 individuals studied, $\sim 14 \%$ subjects should exhibit the loss of B73.1/Leu11c epitope. However, the epitope loss was detected only in 12 subjects (3.5\%). The sequence of the CD16 coding FcyRIIIa gene of the patients with the epitope loss was also analyzed in comparison to healthy control subjects. The sequence analysis of the Fc $\gamma$ RIII $a$ gene revealed that in all patients and healthy subjects, $\mathrm{L}$ was present at amino acid position 48 . These results indicate that the Fc $\gamma$ RIIIa-48 polymorphism has no linkage with the CD16 B73.1/Leu11c mAbs binding capacity in the studied patients. Moreover, analyzing the clinical characteristics of analyzed patients, the correlation of the Fc $\gamma$ RIIIa- $48 \mathrm{R} / \mathrm{H}$ polymorphism with frequent herpes virus infections was not clearly associated. Other studies $(8,22-24)$ postulated the linkage between the loss of B73.1/Leu11c epitope, the presence of Fc $\gamma$ RIIIa-48 $\mathrm{R}$ or $\mathrm{H}$ isoforms and recurrent 
herpes viruses infections in one subject or siblings while in the present study, the group of 12 unrelated patients with the CD16 epitope loss was genotyped. However, though the group of analyzed patients did suffer from recurrent infections, there has been only one patient with recurrent Herpes simplex infections. Nevertheless, the cytotoxic activity of NK cells of the patients has not been analyzed and that is why it cannot be excluded that the activity of NK cells in patients with the epitope loss was diminished.

The sequence analysis of the entire Fc $\gamma$ RIII coding region did not reveal any other changes in the nucleotide sequence that could be responsible for the B73.1/Leu11c epitope loss. Moreover, four other polymorphisms were detected in patients and control subjects, including the changes of amino acids 88, 129, 137 and 158 (Table III). The detected Fc $\gamma$ RIIIa$158 \mathrm{~V} / \mathrm{V}$ isoform was more frequent $(69.6 \%)$ than described previously in Caucasians (20.5\%) (10), yet it could be due to the relatively small group of genotyped subjects. Furthermore, in contrast to other findings that linked the Fc $\gamma$ RIIIa-158F polymorphism to $48 \mathrm{~L}$ isoform and $158 \mathrm{~V}$ with $48 \mathrm{R}$ or $48 \mathrm{H}$ $(10,12)$, no linkage of Fc $\gamma$ RIIIa-48 and -158 genotypes was detected, as all subjects with Fc $\gamma$ RIIIa-158V/V were also Fc $\gamma$ RIIIa-48L/L. In seven subjects (P11 and C5-8, 10-11) the Fc $\gamma$ RIIIa-158 polymorphism was not identified due to the heterozygotic $\mathrm{T} / \mathrm{G}$ isoform detected at 526 nucleotide. However, it can be assumed to be a result of the presence of two different nucleotides at the equivalent position in FcyRIIIa

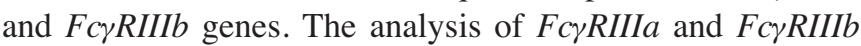
gene sequence (Entrez Gene database, gene ID: 2215) and possible binding of primers used in this study to the $F_{c} \gamma$ RIIIb gene indicate that in the case of nucleotide positions a precise identification of polymorphism was not performed. Both genes are highly similar and in the coding region differ only in some nucleotides. The nucleotide differences occur at the corresponding positions in both genes and at those positions a nucleotide in one gene could be substituted by another that is most frequently present at the same position in the second gene. That is why, in case of these subjects, the 158 amino acid polymorphism in the Fc $\gamma$ RIIIa receptor was marked as indeterminable. The same situation occurred with seven other FC $\gamma$ RIIIa polymorphisms at nucleotides 107, 114, 194, 274, 440, 472, 498, and amino acid positions 18, 20, 47, 64, 129, 137, 148, respectively (Fig. 4). These observations indicate that the method of DNA sequencing under the specific conditions applied in this study and other studies $(8,10,12,20)$ of the FcrRIIIa gene could give ambiguous results not allowing complete determination of the nucleotide and amino acid at every polymorphic site of the Fc $\gamma$ RIIIa receptor.

In summary, our study suggests that the CD16 B73.1/ Leu11c epitope loss may be preferentially associated with the primary immunodeficiency diseases, but not with the occurrence of gastrointestinal malignancies. What is more, the epitope loss seems not to be associated with recurrent herpes viruses infections. Since the anti-CD16 B73.1/ Leu11c $\mathrm{mAb}$ is commonly used in routine immunodiagnostic procedures, the disturbed binding of the mAbs may contribute to low number of NK cells determined and may not enable to detect any of $\mathrm{CD} 14^{+} \mathrm{CD} 16^{++}$monocytes. Therefore, it is advisable to apply additional staining with different clones of the anti-CD16 mAbs in patients with the suspected B73.1/
Leu11c epitope loss. The cause of the epitope loss on NK cells and monocytes remains unsolved after sequence analysis of the entire coding region of $F c \gamma$ RIII $a$ gene. However, the results of our study clearly indicate that this defect does not result from the change of the Fc $\gamma$ RIIIa genotype. This conclusion may explain the NK cell defects and why the B73.1/Leu11c epitope loss does not occur as frequent as the Fc $\gamma$ RIIIa-48R/H polymorphism. Nevertheless, we can only suspect, that the changes of the spatial structure of the receptor in the selected group of patients is responsible for a lack of binding of $\mathrm{B} 73.1 / \mathrm{Leu} 11 \mathrm{c} \mathrm{mAb}$. This change of the structure of the CD16a protein could result from different pattern of glycosylation of the receptor. However, further studies are required to solve this issue.

In summary, this study, based on analysis of the large group of individuals, revealed that disturbed binding of B73.1/Leu11c mAb may be associated with primary immunodeficiency diseases, but not with the Fc $\gamma$ RIIIa-48 polymorphism.

\section{Acknowledgments}

This work was supported by the National Committee for Scientific Research grant No. 3220/PO1/2007/32 and grant No. 2P05C 00129.

\section{References}

1. Ravetch JV and Perussia B: Alternative membrane forms of Fc gamma RIII(CD16) on human natural killer cells and neutrophils. Cell type-specific expression of two genes that differ in single nucleotide substitutions. J Exp Med 170: 481-497, 1989.

2. Passlick B, Flieger D and Ziegler-Heitbrock HWL: Identification and characterization of a novel monocyte subpopulation in human peripheral blood. Blood 74: 2527-2534, 1989.

3. Van de Winkel JG and Capel PJ: Human IgG Fc receptor heterogeneity: molecular aspects and clinical implications. Immunol Today 14: 215-221, 1993.

4. Robertson MJ and Ritz J: Biology and clinical relevance of human natural killer cells. Blood 76: 2421-2438, 1990.

5. Van de Winkel JG and Anderson CL: Biology of human immunoglobulin G Fc receptors. J Leukoc Biol 49: 511-524, 1991.

6. Cooper MA, Fehniger TA, Turner SC, et al: Human natural killer cells: a unique innate immunoregulatory role for the CD56(bright) subset. Blood 97: 3146-3151, 2001.

7. Tamm A and Schmidt RE: The binding epitopes of human CD16 (Fc gamma RIII) monoclonal antibodies. Implications for ligand binding. J Immunol 157: 1576-1581, 1996.

8. De Vries E, Koene HR, Vossen JM, et al: Identification of an unusual Fc $\gamma$ receptor IIIa (CD16) on natural killer cells in a patient with recurrent infections. Blood 88: 3022-3027, 1996.

9. Peltz GA, Grundy HO, Lebo RV, Yssel H, Barsh GS and Moore KW: Human Fc $\gamma$ RIII: Cloning, expression and identification on of the chromosomal locus of two Fc receptors for IgG. Proc Natl Acad Sci USA 86: 1013-1017, 1989.

10. De Haas M, Koene HR, Kleijer M, et al: A triallelic Fc $\gamma$ receptor type IIIA polymorphism influences the binding of human IgG by NK cell Fc-gamma-RIIIA. J Immunol 156: 2948-2955, 1996.

11. Hibbs ML, Tolvanen M and Carpén O: Membrane-proximal Iglike domain of Fc $\gamma$ RIII (CD16) contains residues critical for ligand binding. J Immunol 152: 4466-4474, 1994.

12. Koene HR, Kleijer M, Algra J, Roos D, von dem Borne AE and de Haas M: Fc gammaRIIIa-158V/F polymorphism influences the binding of IgG by natural killer cell Fc gammaRIIIa, independently of the Fc gammaRIIIa-48L/R/H phenotype. Blood 90: 1109-1114, 1997.

13. Wu J, Edberg JC, Redecha PB, et al: A novel polymorphism of Fc $\gamma$ RIIIA (CD16) alters receptor function and predisposes to autoimmune disease. J Clin Invest 100: 1059-1070, 1997. 
14. Koene HR, Kleijer M, Swaak AJ, et al: The Fc-gamma-RIIIA$158 \mathrm{~F}$ allele is a risk factor for systemic lupus erythematosus. Arthritis Rheum 41: 1813-1818, 1998.

15. Seligman VA, Suarez C, Lum R, et al: The Fegamma receptor IIIA-158F allele is a major risk factor for the development of lupus nephritis among Caucasians but not non-Caucasians. Arthritis Rheum 44: 618-625, 2001.

16. Morgan AW, Keyte VH, Babbage SJ, et al: Fc $\gamma$ RIIIA-158V and rheumatoid arthritis: a confirmation study. Rheumatology 42 : 528-533, 2003

17. Kastbom A, Ahmadi A, Söderkvist P and Skogh T: The 158V polymorphism of Fc gamma receptor type IIIA in early rheumatoid arthritis: increased susceptibility and severity in male patients (the Swedish TIRA project). Rheumatology 44: 1294-1298, 2005.

18. Cartron G, Dacheux L, Salles G, et al: Therapeutic activity of humanized anti-CD20 monoclonal antibody and polymorphism in IgG Fc receptor Fc-gamma-RIIIa gene. Blood 99: 754-758, 2002 .

19. Dall'Ozzo S, Tartas S, Paintaud G, et al: Rituximab-dependent cytotoxicity by natural killer cells: influence of FCGR3A polymorphism on the concentration-effect relationship. Cancer Res 64: 4664-4669, 2004.

20. Treon SP, Hansen M, Branagan AR, et al: Polymorphisms in FcyRIIIA (CD16) receptor expression are associated with clinical response to rituximab in Waldenström's macroglobulinemia. J Clin Oncol 23: 474-481, 2005.

21. Musolino A, Naldi N, Bortesi B, et al: Immunoglobulin G fragment $\mathrm{C}$ receptor polymorphisms and clinical efficacy of trastuzumab-based therapy in patients with HER-2/neu-positive metastatic breast cancer. J Clin Oncol 26: 1789-1796, 2008.
22. Jawahar S, Moody C, Chan M, Finberg R, Geha R and Chatila T: Natural killer (NK) cell deficiency associated with an epitopedeficient Fc receptor type IIIA (CD16-II). Clin Exp Immunol 103: 408-413, 1996.

23. Forbes LR, Paessler M and Orange JS: CD16 deficiency discovered in a cohort of patients with severe herpesviral infections using a new diagnostic test. J Allergy Clin Immunol 123: S67(abs. 245), 2009.

24. Kankirawatana S, Dai Y and Atkinson P: A six year old girl with reccurent varicella infections associated with a mutation in CD16 (FCGR3A, Fc $\gamma$ RIIIa). World Allergy Organiz J 134: abst. 420, 2007.

25. Conley ME, Nortarangelo LD and Etzioni A: Diagnostic criteria for primary immunodeficiencies. Clin Immunol 93: 190-197, 1999.

26. Walker A, Kemp A, Hill D and Shelton M: Features of transient hypogammaglobulinemia in infants screened for immunological abnormalities. Arch Dis Child 70: 183-186, 1994.

27. Strayer DR, Carter WA and Brodsky I: Familial occurrence of breast cancer is associated with reduced natural killer cytotoxicity. Breast Cancer Res Treat 7: 187-192, 1986.

28. Bonilla FA, Bernstein IL, Khan DA, et al: Practice parameter for the diagnosis and management of primary immunodeficiency. Ann Allergy Asthma Immunol 94: 1-63, 2005.

29. Anderson CL, Looney RJ, Culp DJ, et al: Human alveolar and peritoneal macrophages bear three distinct classes of $\mathrm{Fc}$ receptors for IgG. J Immunol 145: 196-201, 1990.

30. Weisenseel P, Martin S, Partscht K, Messer G and Prinz JC: Relevance of the low-affinity type of the Fcgamma-receptor IIIa-polymorphism in bullous pemphigoid. Arch Dermatol Res 299: 163-164, 2007. 